\title{
Biomechanical comparison of the K-ROD and Dynesys dynamic spinal fixator systems - A finite element analysis
}

\author{
Hung-Ming Lin ${ }^{\text {a }}$, Yung-Ning Pan ${ }^{\mathrm{a}}$, Chien-Lin Liu ${ }^{\mathrm{b}}$, Li-Ying Huang $^{\mathrm{c}}$, Chang-Hung Huang ${ }^{\mathrm{d}}$ \\ and Chen-Sheng Chen ${ }^{\mathrm{e}, *}$ \\ ${ }^{a}$ Department of Mechanical Engineering, National Taiwan University, Taipei, Taiwan \\ ${ }^{\mathrm{b}}$ Department of Orthopedic Surgery, Taipei Veterans General Hospital, Taipei, Taiwan \\ ${ }^{\mathrm{c}}$ Department of Plastic and Reconstructive Surgery, Chang Gung Memorial Hospital, Taipei, Taiwan \\ ${ }^{\mathrm{d}}$ Department of Biomedical Research, Mackay Memorial Hospital, Tamshui Taipei County, Taiwan \\ ${ }^{\mathrm{e}}$ Department of Physical Therapy and Assistive Technology, National Yang-Ming University, Taipei, \\ Taiwan
}

Received 4 March 2013

Accepted 22 May 2013

\begin{abstract}
Dynamic spinal fixators, such as the Dynesys (DY) and K-ROD (KD) systems, are designed to restore spinal stability and to provide flexibility. The long-term complications of implant breakage and the biomechanics of the adjacent and the bridged levels using the KD system are still unknown. Therefore, this study aims to investigate and compare the biomechanical effects of the KD system and the DY system. Finite element (FE) models of the degenerated lumbar spine, the DY system, and the KD system were each reconstructed. Hybrid-controlled analysis was applied in the three FE models. The FE results indicated that the KD system supplies the most stiffness during extension and the least stiffness during flexion, in contrast to the DY system. In contrast to the DY system, the KD system increased the facet contact force of the adjacent level, but this system decreased the screw stress on the cranial adjacent disc and the pedicle during flexion.
\end{abstract}

Keywords: Finite element analysis, biomechanics, dynamic spinal stabilization devices, K-ROD system, Dynesys

\section{Introduction}

Disc degeneration may induce chronic low-back pain or spinal instability [1,2]. Spinal fusion surgeries are often used to manage spinal instability [3]. However, spinal fusion surgeries have other problems, including donor place ailment, the morbidity of the surgery, and adjacent segment disease $[4,5]$. Several clinical reports have shown that spinal fusion surgery may accelerate degeneration at adjacent segments [6,7]. Consequently, several flexible posterior spinal fixation systems have gradually been introduced in clinics $[8,9]$. These implants are designed to restore spinal stability and reduce the load on the adjacent

\footnotetext{
*Address for correspondence: Chen-Sheng Chen, PhD, Department of Physical Therapy and Assistive Technology, National Yang-Ming University, 155, Sec. 2, Li-Nung St., Taipei, Taiwan 112. Tel.: +886 228267353; Fax: +886 2 28270140; E-mail: cschen@ym.edu.tw.
} 
disc. The Dynesys (DY) system (Zimmer, Minneapolis, MN) is one type of dynamic stabilization device and has been used in clinics for more than a decade. The DY system consists of polycarbonate urethane (PCU) spacers, polyethylene terephthalate (PET) cords, and titanium alloy pedicle screws. The length of the PCU spacers can be adapted for the clinical situation. The PET cords are introduced through the screw heads. The PCU spacers are interposed and fixed after pre-loading $[9,10]$. Moreover, a previous clinical study [11] indicated that the DY system results in satisfactory outcomes, with patient satisfaction remaining as high as $95 \%$. However, complications, including slight screw loosening ( 3 of 26 patients), adjacent segment degeneration (47\% patients), and screw breakage with low back pain (one patient), were also observed at the 4-year follow-up. Biomechanically, the DY system reduces the range of motion (ROM) of the intact spine [12-15], but the DY system is more flexible than a rigid internal fixator [16]. Niosi et al. [14,17] indicated that the DY system with long spacers typically causes an increase in the ROM and a decrease in facet loads compared with the system with short spacers. In addition to the DY system, the K-ROD (KD) system (Paonan Biotech Co., Ltd.) is another type of dynamic stabilization device that has been used in clinics. The KD system is composed of titanium alloy pedicle screws, two titanium alloy cable rods, and two polyaryletherketone (PEEK) spacers. However, the biomechanics of the adjacent and the bridged levels using the KD system are still unknown. Therefore, the purpose of this study is to evaluate the KD system in comparison to the DY system using finite element (FE) models.

\section{Materials and methods}

\subsection{Validation of the FE model}

A three-dimensional nonlinear FE model of the human lumbar spine was created using the commercial software ANSYS 11.0 (ANSYS Inc., Canonsburg, PA, USA) in our previous studies [18,19]. Much of detailed description for this lumbar spine FE model had been reported $[19,20]$. However, the previous FE model did not consider degenerated discs. Therefore, this study varied the material properties of degenerated discs and evaluated the range of motion (ROM) and annulus stress of a degenerated lumbar spine. Umehara et al. [21] reported that the Young's modulus of the ground substance of the annulus increased as the disc degenerated, thus the corresponding Young's modulus, E, of the degenerated discs in each test was increased by $10 \%$ from that of the intact disc. This FE model extracted the L4-L5 motion segment from the entire lumbar spine model to test the degenerated disc. The degenerated disc was simulated with hyperelasticity behavior, and the material properties were controlled using two parameters $(\mathrm{C} 1, \mathrm{C} 2)$ in the Mooney-Rivlin formulation. The degenerated L4-L5 model was validated by experimental results [22] by measuring the ROM using a 7.5 Nm moment for flexion-extension, lateral bending, and torsion. To examine any discrepancies, the FE analysis was compared with a previous in vitro test [22] using the following equation:

$$
\begin{aligned}
& \text { error } \\
& \quad=\sqrt{\left(\mathrm{ROM}_{\mathrm{P}}-\mathrm{ROM}_{\mathrm{E}}\right)_{\text {flexion}+ \text { extension }}^{2}+\left(\mathrm{ROM}_{\mathrm{P}}-\mathrm{ROM}_{\mathrm{E}}\right)_{\text {lateral bending }}^{2}+\left(\mathrm{ROM}_{\mathrm{P}}-\mathrm{ROM}_{\mathrm{E}}\right)_{\text {torsion }}^{2},},
\end{aligned}
$$

$\mathrm{ROM}_{\mathrm{P}}$ : range of motion in the present $\mathrm{FE}$ study, $\mathrm{ROM}_{\mathrm{E}}$ : range of motion in the previous in vitro test. 
The FE analysis exhibited a similar trend to that of the previous experimental results, as listed in Table 1. When the Young's modulus of the annulus fibrosis of the disc was increased by $100 \%$ (the corresponding Young's modulus E was $6.94 \mathrm{MPa}$ ), the ROM of L4-L5 was closer to that of the in vitro results. The error was smallest (0.72) after applying a data normalization method.

To validate the distribution of the disc stress, this study applied a compression force of $2000 \mathrm{~N}$ at the top of the L4 vertebral body and fixed all nodes at the bottom surface of the L5 vertebral body. This FE analysis calculated the disc annulus stress from the posterior to anterior direction on the disc. These results indicated that the disc annulus stress distribution showed a similar tendency in this study to that of the in vitro experiment [23] (Fig. 1). However, the stress in the FE analysis was lower than that in experimental result because the cross-sectional area of the disc in the FE model was greater.

Afterward, an entire lumbar spine model with degenerative disc disease (DDD) was created to use with either the DY model or the KD model using the above parameters (disc annulus fibrosis: $\mathrm{C} 1=0.84$,

Table 1

Comparison of FE results and the in vitro testing

\begin{tabular}{|c|c|c|c|c|c|c|c|}
\hline \multirow[t]{2}{*}{ Various (\%) } & \multicolumn{3}{|c|}{ ROM of the present study } & \multicolumn{3}{|c|}{ ROM of the in vitro testing [22] } & \multirow[t]{2}{*}{ Error } \\
\hline & Flexion-extension & Lateral bending & Torsion & Flexion-extension & Lateral bending & Torsion & \\
\hline$\uparrow 10 \%$ & 7.38 & 8.48 & 4.68 & $6.16 \pm 4.10$ & $6.01 \pm 3.49$ & $3.71 \pm 2.11$ & 2.92 \\
\hline$\uparrow 20 \%$ & 7.15 & 8.14 & 4.60 & & & & 2.51 \\
\hline$\uparrow 30 \%$ & 6.92 & 7.82 & 4.54 & & & & 2.13 \\
\hline$\uparrow 40 \%$ & 6.72 & 7.54 & 4.46 & & & & 1.79 \\
\hline$\uparrow 50 \%$ & 6.53 & 7.30 & 4.40 & & & & 1.51 \\
\hline$\uparrow 60 \%$ & 6.35 & 7.08 & 4.34 & & & & 1.26 \\
\hline$\uparrow 70 \%$ & 6.18 & 6.88 & 4.28 & & & & 1.04 \\
\hline$\uparrow 80 \%$ & 6.03 & 6.74 & 4.24 & & & & 0.91 \\
\hline$\uparrow 90 \%$ & 5.88 & 6.56 & 4.18 & & & & 0.78 \\
\hline$\uparrow 100 \%$ & 5.74 & 6.40 & 4.14 & & & & 0.72 \\
\hline
\end{tabular}
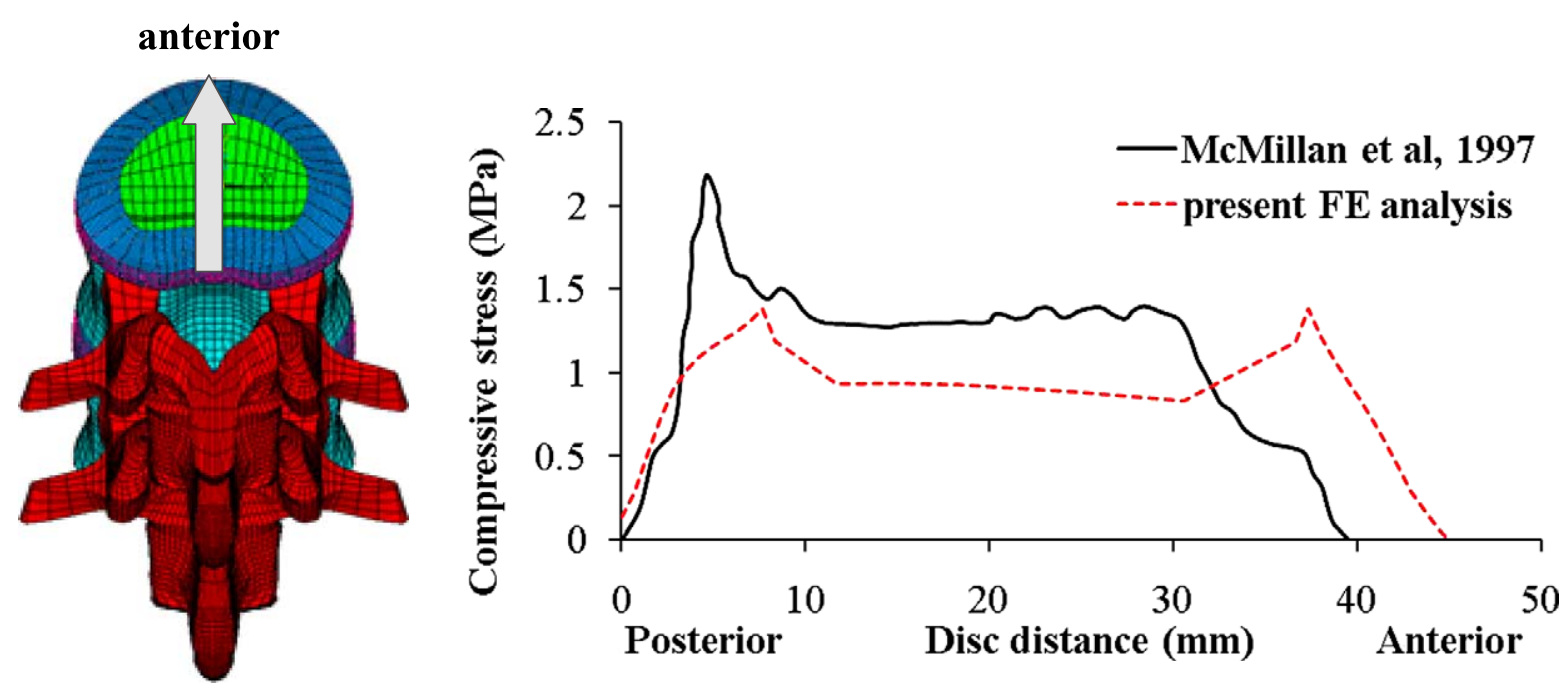

Fig. 1. Comparison between the in vitro test and the FE analysis of the disc stress from the posterior to the anterior region. (Colors are visible in the online version of the article; http://dx.doi.org/10.3233/BME-130766.) 

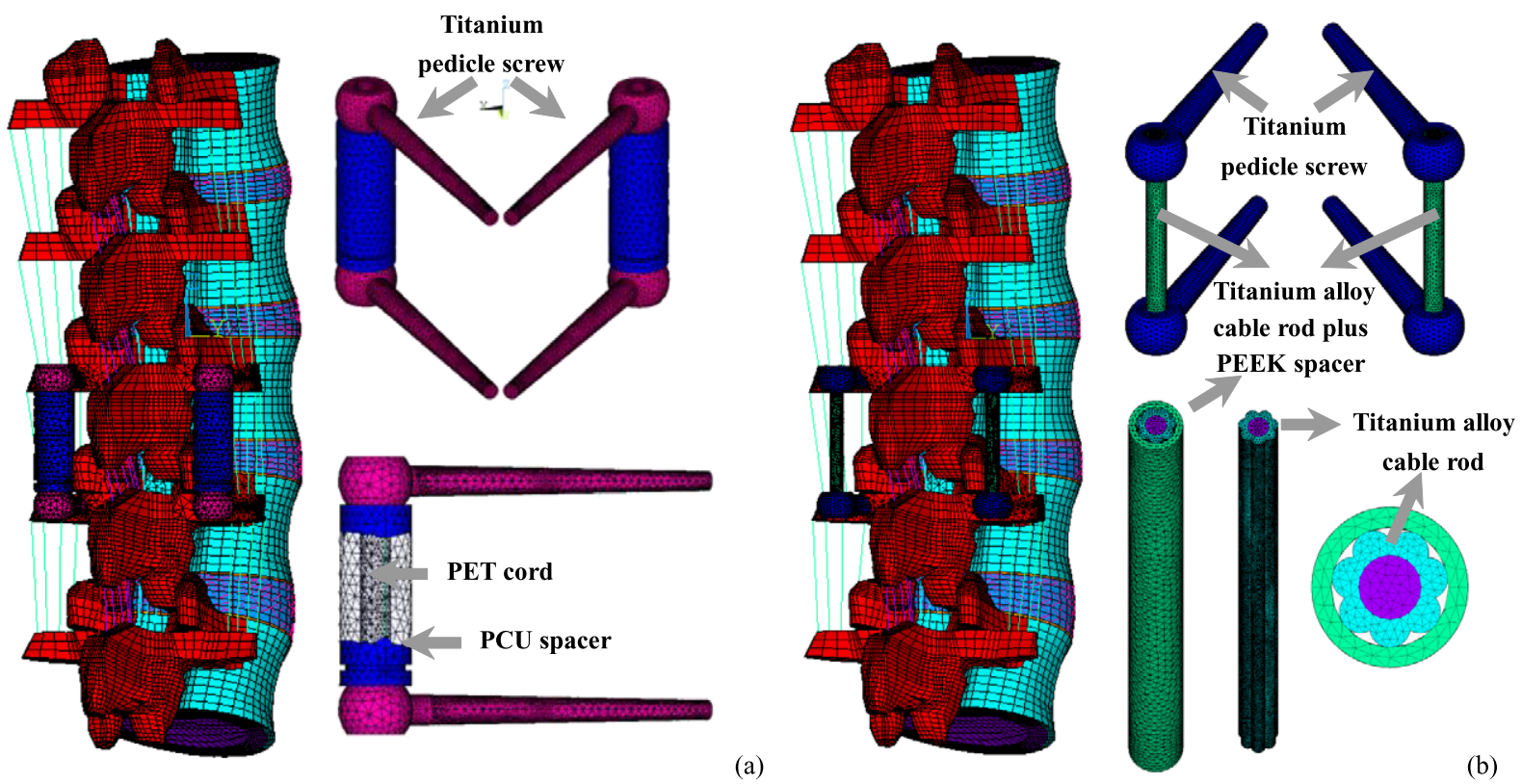

Fig. 2. FE model: (a) DY system (b) KD system. (Colors are visible in the online version of the article; http://dx.doi.org/ 10.3233/BME-130766.)

$\mathrm{C} 2=0.21$ and Young's modulus $\mathrm{E}=6.94 \mathrm{MPa}$; nucleus: elastic modulus of 1.66 MPa and Poisson's ratio of 0.499 ) to simulate the stabilization of grade II disc degeneration. However, the DDD model, which consisted of 112,174 elements and 94,162 nodes, did not include the spinal fixation system.

\subsection{FE model of the DY and KD systems}

The DY [18,24] and the KD models (Fig. 2) were bilaterally inserted into the L3-L4 level of the model. The implant for the DY and the KD models consisted of four titanium alloy screws (diameter: $6.4 \mathrm{~mm}$, length: $45 \mathrm{~mm}$ ). Moreover, two PCU spacers (diameter: $12 \mathrm{~mm}$, length: $30 \mathrm{~mm}$ ) and two PET cords, which contacted the screw in DY model, were modeled. In the KD FE model, two titanium alloy cable rods $(\varnothing 3 \mathrm{~mm} \times \mathrm{L} 46 \mathrm{~mm})$ and two PEEK spacers $(\varnothing 4 \mathrm{~mm} \times \mathrm{L} 46 \mathrm{~mm})$ were modeled. The DY model consisted of 292,502 elements and 126,090 nodes. The KD model included 565,068 elements and 192,750 nodes. The material properties of the implant were based on the information from a previous studies [18,24,25].

\subsection{Boundary and loading conditions}

Several studies indicated that controlling the same ROM is a reasonable approach for predicting the effects of the implanted spinal instrumentation on the adjacent levels $[20,26,27]$. Therefore, this research implemented a hybrid-controlled analysis. A moment was applied to the top surface of the L1 vertebral body. All nodes pertaining to the bottom surface of the L5 vertebral body were fixed. Moreover, ROMs under flexion, extension, torsion, and lateral bending were controlled by $12^{\circ}, 10^{\circ}, 8^{\circ}$ and $20^{\circ}$, respectively. All of the loading conditions are listed in Table 2. 
Table 2

Comparison of ROM for each motion segment in three FE models

\begin{tabular}{lccccccc}
\hline Motion & Model & $\begin{array}{c}\text { L1-L2 } \\
(\text { degree })\end{array}$ & $\begin{array}{c}\text { L2-L3 } \\
(\text { degree })\end{array}$ & $\begin{array}{c}\text { L3-L4 } \\
(\text { degree })\end{array}$ & $\begin{array}{c}\text { L4-L5 } \\
(\text { degree })\end{array}$ & $\begin{array}{c}\text { Moment } \\
(\text { Nm })\end{array}$ & $\begin{array}{c}\text { L1-L5 } \\
\text { Stiffness } \\
(\text { Nm/degree })\end{array}$ \\
\hline Flexion & DDD & 2.54 & $2.82(100 \%)$ & $2.94(100 \%)$ & 3.75 & 9.97 & $0.83(100 \%)$ \\
& DY & 3.42 & $3.63(129 \%)$ & $0.06(2 \%)$ & 4.87 & 14.60 & $1.22(147 \%)$ \\
Extension & KD & 3.16 & $3.34(118 \%)$ & $0.88(30 \%)$ & 4.51 & 13.18 & $1.11(133 \%)$ \\
& DDD & 2.30 & $2.35(100 \%)$ & $2.27(100 \%)$ & 3.14 & 12.47 & $1.24(100 \%)$ \\
& DY & 2.36 & $2.39(102 \%)$ & $1.90(84 \%)$ & 3.32 & 13.18 & $1.32(107 \%)$ \\
Lateral bending & KD & 2.86 & $2.83(120 \%)$ & $0.78(34 \%)$ & 3.53 & 16.67 & $1.68(135 \%)$ \\
& DDD & 4.11 & $4.60(100 \%)$ & $5.05(100 \%)$ & 6.14 & 26.57 & $1.33(100 \%)$ \\
Torsion & DY & 4.69 & $5.23(114 \%)$ & $3.05(60 \%)$ & 6.98 & 30.17 & $1.51(114 \%)$ \\
& KD & 4.88 & $5.29(115 \%)$ & $2.74(54 \%)$ & 7.17 & 31.77 & $1.58(119 \%)$ \\
& DDD & 1.60 & $1.75(100 \%)$ & $2.07(100 \%)$ & 2.35 & 12.13 & $1.56(100 \%)$ \\
& DY & 1.72 & $1.84(105 \%)$ & $1.83(88 \%)$ & 2.55 & 14.20 & $1.79(115 \%)$ \\
& KD & 1.81 & $1.85(106 \%)$ & $1.61(78 \%)$ & 2.55 & 14.44 & $1.85(119 \%)$ \\
\hline
\end{tabular}

Note: The parentheses indicate the following value: $\frac{\text { DDD,DY or KD }}{\text { DDD }} \times 100 \%$.

\section{Results}

\subsection{ROM of the lumbar spine}

The KD and the DY models provided stabilization at the bridged level. The ROMs from the bridged level for all motions of the KD and the DY models were at most 0.78 and 0.88 times those of the DDD model (Table 2). The flexion of the KD model was greater than that of the DY model. The stiffnesses of the KD and the DY models were at most 1.35 and 1.15 times the stiffnesses of the DDD model in extension, lateral bending and torsion.

\subsection{Stress of the adjacent disc and the lumbar spine}

The cranial adjacent disc stresses (CADS) of the KD and the DY model were 1.29 and 1.02 times the L2-L3 disc stress of the DDD model (Table 3) in extension. However, the KD model had lower CADS than the DY model in flexion. The CADS from the KD model was similar to the CADS in the DY model in lateral bending and torsion. The differences between those models were within $5 \%$.

Additionally, the KD model decreased the region of higher stress at the CADS in flexion relative to the same region in the DY model (Fig. 3). In contrast, the stress distribution of the posterior bony element in flexion was similar in the KD and DDD models (Fig. 4). The KD model alleviated the higher stress range from the L3-L4 posterior bony element, especially in the pedicle and the posterior bony element around the implant, compared with the corresponding range in the DY model (Fig. 4).

\subsection{Facet contact forces $(F C F)$}

The KD model increased the L2-L3 FCF and decreased the L3-L4 FCF compared with the corresponding values in the DY model for all motions. The L2-L3 FCF of the KD model was at most 2.19 times that of the DDD model and 0.33 times the L3-L4 FCF of the DDD model as well (Table 4). 
Table 3

Comparison of disc stress in three FE models

\begin{tabular}{lccc}
\hline Motion & Model & L2-L3 & L3-L4 \\
\hline Flexion & DDD & $792(100 \%)$ & $737(100 \%)$ \\
& DY & $1070(135 \%)$ & $282(38 \%)$ \\
Extension & KD & $985(124 \%)$ & $319(43 \%)$ \\
& DDD & $689(100 \%)$ & $645(100 \%)$ \\
Lateral bending & DY & $700(102 \%)$ & $492(76 \%)$ \\
& KD & $886(129 \%)$ & $328(51 \%)$ \\
Torsion & DDD & $1590(100 \%)$ & $1570(100 \%)$ \\
& DY & $1910(120 \%)$ & $1060(68 \%)$ \\
& KD & $1980(125 \%)$ & $820(52 \%)$ \\
& DDD & $386(100 \%)$ & $407(100 \%)$ \\
& DY & $451(117 \%)$ & $559(137 \%)$ \\
\hline
\end{tabular}

Notes: Unit $-\mathrm{kPa}$. The parentheses indicate the following value: $\frac{\mathrm{DDD}, \mathrm{DY} \text { or } \mathrm{KD}}{\mathrm{DDD}} \times 100 \%$.

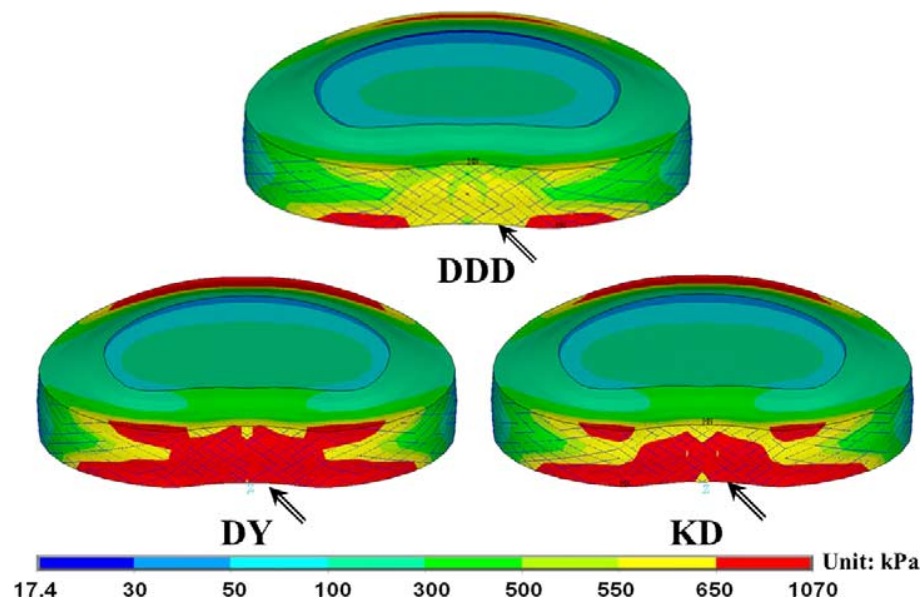

Fig. 3. The stress distribution of the cranial adjacent disc in L2-L3 was a von Mises stress distribution in flexion. The arrowhead signifies the range of the higher stress distribution. (Colors are visible in the online version of the article; http://dx.doi.org/ 10.3233/BME-130766.)

\subsection{Pedicle screw stress}

In flexion, the pedicle screw of the KD model exhibited lower stress than that of the DY model (Fig. 5). The maximum screw stress of the KD model was 0.68 times that of the DY model. In extension and lateral bending, the screw stress distribution did not noticeably differ. The maximum stress of the pedicle screw was within $0.55 \%$ of the stresses of the KD and DY model in lateral bending. However, the maximum stress in extension for the KD model was 0.83 times that of the DY model. Regarding torsion, the KD model demonstrated greater screw stress than the DY model. The maximum screw stress of the KD model was 1.62 times that of the DY model. 


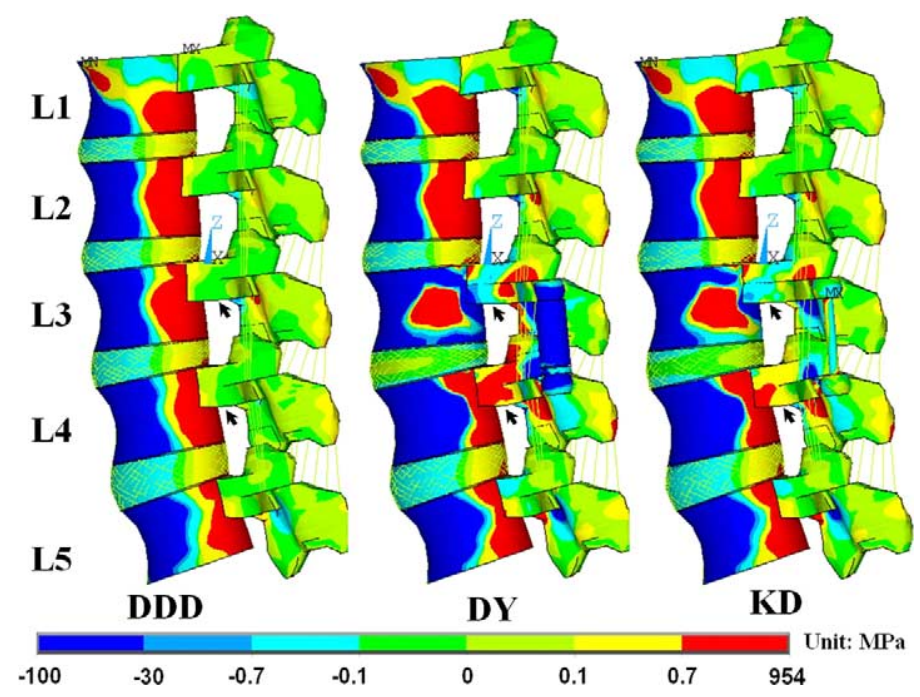

Fig. 4. Compressive and tensile stress distribution of overall lumbar spine when under flexion. The small arrowhead indicates that the stress distribution from the KD system is smaller than that from the DY model in the pedicle. Note: Positive values represent tensile stress. Negative values represent compressive stress. (Colors are visible in the online version of the article; http://dx.doi.org/10.3233/BME-130766.)

Table 4

Comparison of facet joint force in three FE models

\begin{tabular}{lccc}
\hline Motion & Model & L2-L3 & L3-L4 \\
\hline Extension & DDD & $44(100 \%)$ & $87(100 \%)$ \\
& DY & $47(107 \%)$ & $46(53 \%)$ \\
Lateral bending & KD & $72(164 \%)$ & $0.71(1 \%)$ \\
& DDD & $27(100 \%)$ & $31(100 \%)$ \\
Torsion & DY & $29(107 \%)$ & $45(145 \%)$ \\
& KD & $59(219 \%)$ & $1(3 \%)$ \\
& DDD & $147(100 \%)$ & $141(100 \%)$ \\
Notes: Unit - N. The parentheses indicate the following value: $\frac{\text { DDD,DY or KD }}{\text { DDD }} \times 100 \%$.
\end{tabular}

\section{Discussion}

A three-dimensional FE model of a degenerated lumbar spine was used to evaluate both the KD and DY systems at the adjacent and bridged levels. A previous in vitro test [16] indicated that the DY system was able to restore the ROM of the bridged level to that of the intact condition in extension, as measured using this FE analysis. However, in flexion, the DY system exhibited greater stiffness than did the KD system because the DY system had greater cord pretension, allowing it to resist the flexion moment. Additionally, previous studies $[10,28]$ reported that an implant with higher stiffness induced a higher $\mathrm{ROM}$ at the adjacent level and a lower ROM at the bridged level. The ROM from the KD system was less at the adjacent level and greater at the bridged level than that from the PEEK (semirigid) and titanium 

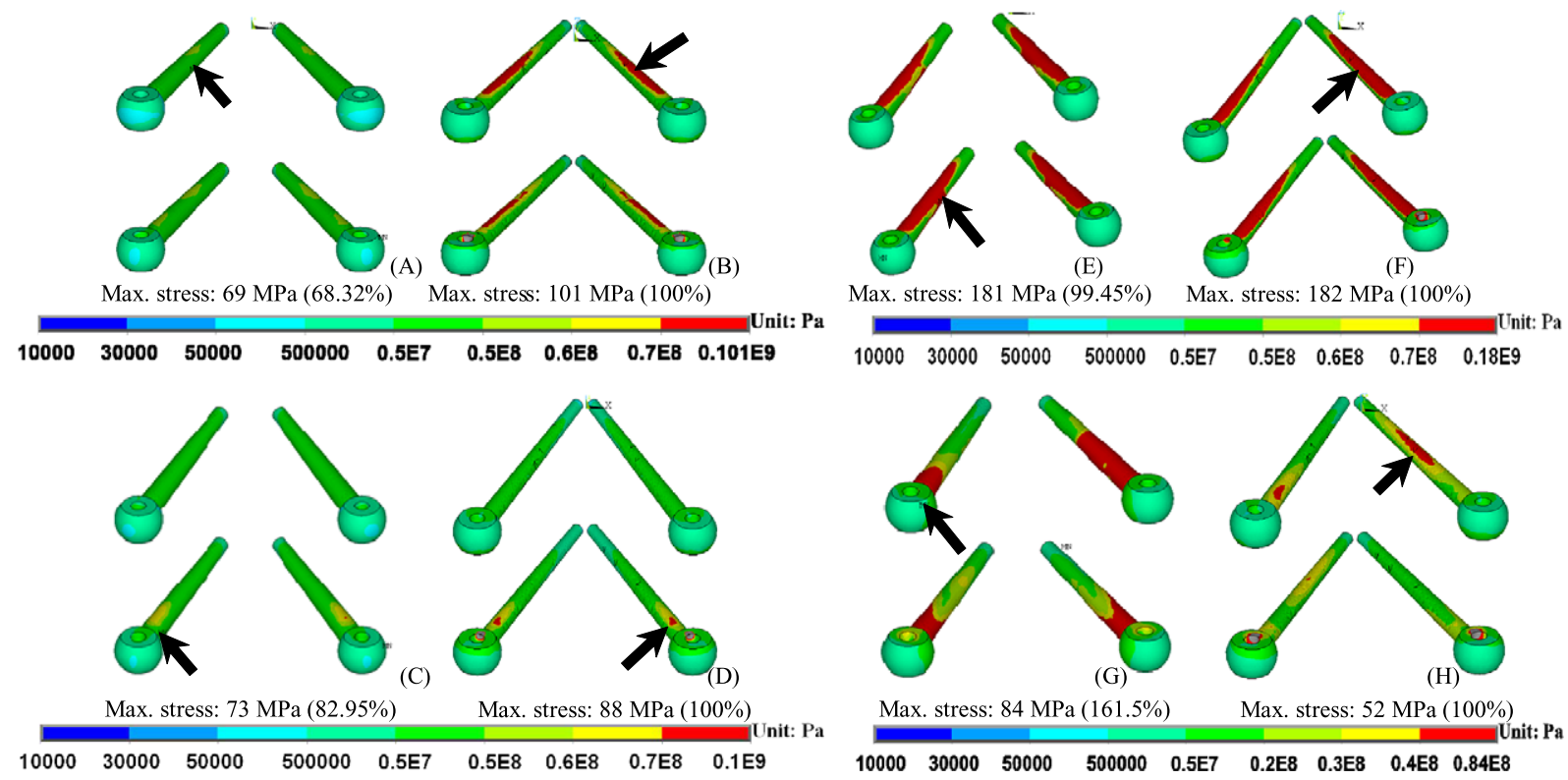

Fig. 5. Screw stress distribution: (A) KD-flexion, (B) DY-flexion, (C) KD-extension, (D) DY-extension, (E) KD-lateral bending, (F) DY-lateral bending, (G) KD-torsion, and (H) DY-torsion. The arrowhead indicates the position of maximum stress. (Colors are visible in the online version of the article; http://dx.doi.org/10.3233/BME-130766.)

systems used in Galbusera's research (Fig. 6) [28]. The earlier in vitro study [29] demonstrated that the DY system induced a remarkable increasing trend in the adjacent disc stress compared with the stress in the intact condition. The present FE results were consistent with those in previous studies [29] as well. However, the stress-shielding effect on the adjacent level from the DY system was still less than that from the rigid-fixation system [10]. In torsion, the CADS and the adjacent ROM from the KD system were similar to those from the DY system because the cord pretension in the DY system provided a greater constraint on the sagittal plane than on the transverse plane. Therefore, there was only a small difference (within 5\%) between the DY and KD systems.

These results indicated that the reduction in the FCF found in the DY system occurred in extension rather than in torsion, and the trend was consistent with the earlier FE study [10]. Both the decrease in the FCF at the bridged level and the increase in the FCF at the adjacent level were larger in the KD system than in the DY system. This result demonstrated that the PEEK rod in the KD system had greater torsional stiffness for resisting the facet joint contact than the PCU spacer in DY system. Therefore, for the KD system, the greater constraint of the facet joint contact at the bridged level resulted in compensation of the excessive facet joint contact at the adjacent level. The results demonstrated that the KD system could increase the adjacent facet loading in all motions in this FE analysis.

For the stress distribution, the KD system could decrease the range of the higher stress distribution around the pedicle in flexion compared with the range of the DY system because of the lower stiffness. Because the PEEK rod was stiffer than the PCU spacer, the KD system absorbed a larger portion of the load on the posterior bony element. Conversely, less stress was transmitted to the posterior bony element in the DY system, resulting in a greater concentration of stress around the pedicle. This phenomenon also induced the pedicle screw in the DY system to receive greater stress than in the KD model (Fig. 5(A), (B)). As a result, screw loosening was found in the clinic in the DY system. Additionally, the 


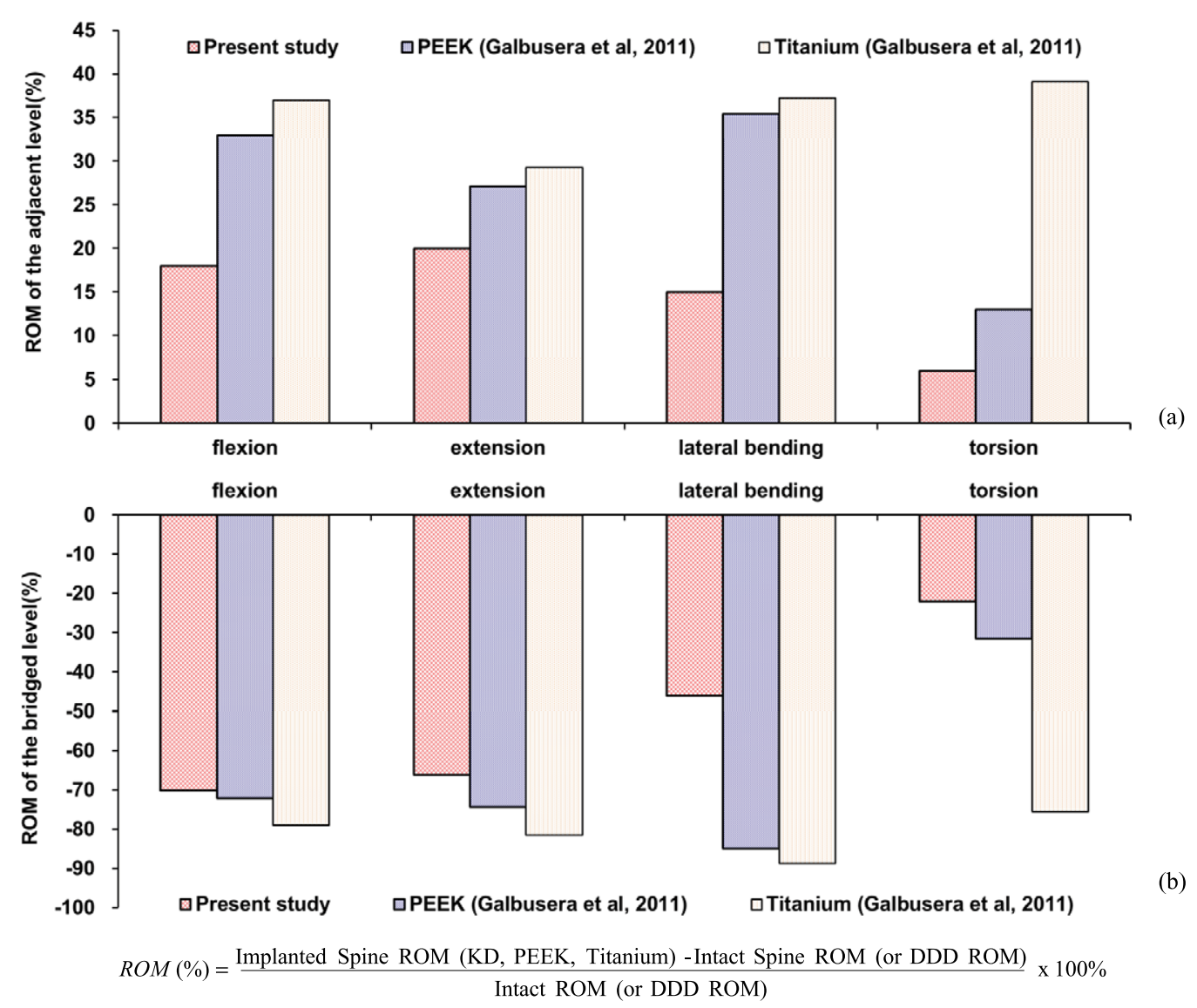

Fig. 6. Comparison of ROM (\%) for the adjacent and bridged segments in the lumbar spine with different spinal instrumentations. (Colors are visible in the online version of the article; http://dx.doi.org/10.3233/BME-130766.)

flexion tendencies in the KD and the DY systems were opposite to that of the DDD model because of increased stiffness. Because the DDD model did not include a spinal implant, the posterior bony element and pedicle exhibited a stress distribution that was almost lower than that in the KD and DY models. Moreover, the DDD model demonstrated a compressive and tensile stress on the vertebral body, but adding a spinal implant also apparently influenced the stress distribution of vertebral body, as shown in Fig. 4. The stiffness of the spinal implant in the posterior bony element was not only associated with the stress distribution of the pedicle but also affected the stress distribution of the vertebral body.

The restrictions of the FE analysis are listed below. The material properties, such as the nonlinear behavior of the spinal ligaments and the viscoelasticity of the disc, were simplified and idealized from those of a cadaveric specimen. The various grades of disc degeneration, such as delamination, dehydration, and reduced disc height, were not taken into account. The thread on the screw was simplified as well. The loading conditions of the FE models were similar to those of the in vitro test. Thus, muscle contractions, complicated external loads, body weight, and movements of the pelvis were not considered in this study. In the rod of the KD model, the seven titanium-alloy cables were separate but still contacted each other. However, this study assumed that the seven titanium-alloy cables were adhered together because the cables were constrained by the PEEK spacer. 


\section{Conclusions}

The KD system supplied greater stiffness in extension, torsion and lateral bending than did the DY system. The KD system also increased the FCF of the adjacent level in all motions. However, the KD system could decrease the CADS, pedicle screw stress, and pedicle stress in flexion, in contrast to the DY system, which did could not. Additionally, the KD and DY systems increased the stiffness to within $47 \%$ of the value in the DDD model in all motions.

\section{Acknowledgements}

This study was supported by the National Science Council (NSC 101-2314-B-075-001-MY3).

\section{References}

[1] S. Roberts, S.M. Eisenstein, J. Menage, E.H. Evans and I.K. Ashton, Mechanoreceptors in intervertebral discs. Morphology, distribution, and neuropeptides, Spine 20 (1995), 2645-2652.

[2] A.C. Schwarzer, C.N. Aprill, R. Derby, J. Fortin, G. Kine and N. Bogduk, The relative contributions of the disc and zygapophyseal joint in chronic low back pain, Spine 19 (1994), 801-806.

[3] T. Kaner, M. Sasani, T. Oktenoglu and A.F. Ozer, Dynamic stabilization of the spine: a new classification system, Turk. Neurosurg. 20 (2010), 205-215.

[4] P. Fritzel, O. Hagg, P. Wessberg and A. Nordwall, Swedish Lumbar Spine Study Group, 2001 Volvo Award Winner in Clinical studies: Lumbar fusion versus nonsurgical treatment for chronic low back pain, Spine 26 (2001), 2521-2534.

[5] J.N.A. Gibson, J.C. Grant and G. Waddel, The cochrane review of surgery for lumbar disc prolapse and degenerative lumbar spondylosis, Spine 24 (1999), 1820-1832.

[6] J.C. Eck, S.C. Humphreys and S.D. Hodges, Adjacent-segment degeneration after lumbar fusion: a review of clinical, biomechanical, and radiologic studies, Am. J. Orthop. 28 (1999), 336-340.

[7] J.D. Schlegel, J.A. Smith and R.L. Schleusener, Lumbar motion segment pathology adjacent to thoracolumbar, lumbar, and lumbosacral fusions, Spine 21 (1996), 970-981.

[8] A. Gardner and K.C. Pande, Graf ligamentoplasty: a 7-year follow-up, Eur. Spine J. 11 (2002), 157-163.

[9] T.M. Stoll, G. Dubois and O. Schwarzenbach, The dynamic neutralization system for the spine: a multi-center study of a novel non-fusion system, Eur. Spine J. 11 (2002), 170-178.

[10] A. Rohlmann, N. Burra, T. Zander and G. Bergmann, Comparison of the effects of bilateral posterior dynamic and rigid fixation devices on the loads in the lumbar spine: a finite element analysis, Eur. Spine J. 16 (2007), 1223-1231.

[11] S. Schaeren, I. Broger and B. Jeanneret, Minimum four-year follow-up of spinal stenosis with degenerative spondylolisthesis treated with decompression and dynamic stabilization, Spine 33 (2008), 636-642.

[12] R. Eberlein, A. Holzapfel and M. Frohlich, Multi-segment FEA of the human lumbar spine including the heterogeneity of the annulus fibrosus, Comput. Mech. 34 (2004), 147-163.

[13] S. Freudiger, G. Dubios and M. Lorrain, Dynamic neutralization of the lumbar spine confirmed on a new lumbar spine simulator in vitro, Arch. Orthop. Trauma Surg. 199 (1999), 127-132.

[14] C.A. Niosi, Q.A. Zhu, D.C. Wilson, O. Keynan, D.R. Wilson and T.R. Oxland, Biomechanical characterization of the three-dimensional kinematic behavior of the Dynesys dynamic stabilization system: an in vitro study, Eur. Spine J. 15 (2006), 913-922.

[15] T. Zander, A. Rohlmann, N.K. Burra and G. Bergmann, Effect of a posterior dynamic implant adjacent to a rigid spinal fixator, Clin. Biomech. 21 (2006), 767-774.

[16] W. Schmoelz, J.F. Huber, T. Nydegger, Dipl-Ing, L. Claes and H.J. Wilke, Dynamic Stabilization of the lumbar spine and its effects on adjacent segments: An in vitro experiment, J. Spinal Disord. Tech. 16 (2003), 418-423.

[17] C.A. Niosi, D.C. Wilson, Q. Zhu, O. Keynan, D.R. Wilson and T.R. Oxland, The effect of dynamic posterior stabilization on facet joint contact forces, Spine 33 (2008), 19-26.

[18] C.L. Liu, Z.C. Zhong, H.W. Hsu, S.L. Shih, S.T. Wang, C. Hung and C.S. Chen, Effect of the cord pretension of the Dynesys dynamic stabilization system on the biomechanics of the lumbar spine: a finite element analysis, Eur. Spine J. 20 (2011), 1850-1858.

[19] S.H. Chen, Z.C. Zhong, C.S. Chen, W.J. Chen and C. Hung, Biomechanical comparison between lumbar disc arthroplasty and fusion, Med. Eng. Phys. 31 (2009), 244-253. 
[20] Z.C. Zhong, S.H. Chen and C. Hung, Load- and displacement controlled finite element analyses on fusion and non-fusion spinal implants, Proc. Inst. Mech. Engr. Part H: J. Eng. Med. 223 (2009), 143-157.

[21] S. Umehara, S. Tadano, K. Abumi, K. Katagiri, K. Kaneda and T. Ukai, Effects of degeneration on the elastic modulus distribution in the lumbar intervertebral disc, Spine 21 (1996), 811-819.

[22] A. Kettler, F. Rohlmann, C. Ring, C. Mack and H.J. Wilke, Do early stages of lumbar intervertebral disc degeneration really cause instability? Evaluation of an in vitro database, Eur. Spine J. 20 (2011), 578-584.

[23] D.W. McMillan, D.S. McNally, G. Garbutt and M.A. Adams, Stress distributions inside intervertebral discs: the validity of experimental "stress profilometry", Proc. Inst. Mech. Eng. H 210 (1996), 81-87.

[24] C.L. Liu, Z.C. Zhong, S.L. Shih, C. Hung, Y.E. Lee and C.S. Chen, Influence of Dynesys system screw profile on adjacent segment and screw, J. Spinal Disord. Tech. 23 (2010), 410-417.

[25] M.F. Chiang, J.M. Teng, C.H. Huang, C.K. Cheng, C.S. Chen, T.K. Chang and S.H. Chao, Finite element analysis of cage subsidence in cervical interbody fusion, Journal of Medical and Biological Engineering 24(4) (2004), 201-208.

[26] V.K. Goel, J.N. Grauer, T.C.H. Patel, A. Biyani, K. Sairyo, S. Vishnubhotla, A. Matyas, I. Cowgill, M. Shaw, R. Long, D. Dick, M. Panjabi and H. Serhan, Effects of charite' artificial disc on the implanted and adjacent spinal segments mechanics using a hybrid testing protocol, Spine 30 (2005), 2755-2764.

[27] M. Panjabi, G. Henderson, C. Abjornson and J. Yue, Multidirectional testing of one- and two-level ProDisc-L versus simulated fusions, Spine 32 (2007), 1311-1319.

[28] F. Galbusera, C.M. Bellini, F. Anasetti, C. Ciavarro, A. Lovi and M. Brayda-Bruno, Rigid and flexible spinal stabilization devices: a biomechanical comparison, Med. Eng. Phys. 33 (2011), 490-496.

[29] W. Schmoelz, J.F. Huber, T. Nydegger, L. Claes and H.J. Wilke, Influence of a dynamic stabilization system on load bearing of a bridged disc: an in vitro study of intradiscal pressure, Eur. Spine J. 15 (2006), 1276-1285. 